\title{
Characterization and stability of approximately dual g-frames in Hilbert spaces
}

Yan-Ling Fu' and Wei Zhang ${ }^{2 *}$

"Correspondence:

zwfylhappy@126.com

${ }^{2}$ School of Mathematics and Information Sciences, Henan

University of Economics and Law Zhengzhou, P.R. China

Full list of author information is available at the end of the article

\begin{abstract}
This paper addresses approximately dual g-frames. First, we establish a connection between approximately dual $\mathrm{g}$-frames and dual $\mathrm{g}$-frames and obtain a characterization of approximately dual $g$-frames. Second, we give results on stability of approximately dual $\mathrm{g}$-frames, which cover the results obtained by other authors.
\end{abstract}

MSC: $42 \mathrm{C} 15 ; 42 \mathrm{C} 40$

Keywords: Frame; g-frame; Dual g-frame; Approximately dual g-frame

\section{Introduction}

The notion of frame dates back to Gabor [1] (1946) and Duffin and Schaeffer [2] (1952). Gabor [1] proposed the idea of decomposing a general signal in terms of elementary signals, and Duffin and Schaeffer [2] abstracted "these elementary signals" as the notion of frame. However, the frame theory had not attracted much attention until the celebrated work by Daubechies, Crossman, and Meyer [3] in 1986. So far, the theory of frame has seen great achievements in pure mathematics, science, and engineering ([4-13]). In 2006, Sun [14] introduced a generalized frame (simply g-frame), which covers all other generalizations of frames, for example, fusion frames [15], bounded quasiprojectors [16], and so on. Now, the research of g-frames has obtained many results [17-19]. This paper addresses approximately dual g-frames in Hilbert spaces.

Recall that a sequence $\left\{f_{i}\right\}_{i \in I}$ in a separable Hilbert space $H$ is a frame if

$$
A_{1}\|f\|^{2} \leq \sum_{i \in I}\left|\left\langle f, f_{n}\right\rangle\right|^{2} \leq B_{1}\|f\|^{2}
$$

for all $f \in \mathcal{H}$ and some positive constants $A_{1}, B_{1}$. Given a frame $\left\{f_{i}\right\}_{i \in I}$, another frame $\left\{h_{i}\right\}_{i \in I}$ is said to be a dual frame of $\left\{f_{i}\right\}_{i \in I}$ if

$$
f=\sum_{i \in I}\left\langle f, f_{i}\right\rangle h_{i}, \quad \forall f \in H,
$$

or, equivalently,

$$
f=\sum_{i \in I}\left\langle f, h_{i}\right\rangle f_{i}, \quad \forall f \in H .
$$

(c) The Author(s) 2018. This article is distributed under the terms of the Creative Commons Attribution 4.0 International License (http://creativecommons.org/licenses/by/4.0/), which permits unrestricted use, distribution, and reproduction in any medium, provided you give appropriate credit to the original author(s) and the source, provide a link to the Creative Commons license, and indicate if changes were made. 
To find the dual frames for a general frame is a fundamental problem in the frame theory. Usually, it is not easy due to involving complicated computation. In 2010, Christensen [20] introduced the notion of approximately dual frames. Bessel sequences $\left\{f_{i}\right\}_{i \in I}$ and $\left\{h_{i}\right\}_{i \in I}$ in a separable Hilbert space $\mathcal{H}$ are said to be approximately dual frames if

$$
\left\|f-\sum_{i \in I}\left\langle f, h_{i}\right\rangle f_{i}\right\| \leq\|f\|, \quad \forall f \in H
$$

or

$$
\left\|f-\sum_{i \in I}\left\langle f, f_{i}\right\rangle h_{i}\right\| \leq\|f\|, \quad \forall f \in H .
$$

In 2014, Khosravi et al. [21] first introduced the notion of approximately dual g-frames, which generalize the usual approximately dual frames. They proved that a pair of operator sequences form approximately dual frames if and only if their induced sequences form a pair of approximately dual g-frames. They also obtained some important properties and applications of approximately dual frames. Later, many results on approximately dual gframes were obtained (see [22, 23]).

Motivated by [21], in this paper, we focus on the characterization and stability of approximately dual g-frames and their connection with dual g-frames. Sect. 2 is an auxiliary one, where we recall some basic notions, properties, and some related results. In Sect. 3, we establish a characterization of approximately dual g-frames and discuss some properties of approximately dual (dual) g-frames. In Sect. 4, we give some stability results of approximately dual g-frames, which cover the results obtained by other authors.

\section{Preliminaries}

We begin with some basic notions and results of g-frames. See $[14,17,18]$ for details.

Given separable Hilbert spaces $H$ and $V$, let $\left\{V_{j}: j \in J\right\}$ be a sequence of closed subspaces of $V$ with $J$ being a subset of integers $\mathbb{Z}$. The identity operator on $H$ is denoted by $I_{H}$. The set of all bounded linear operators from $H$ into $V_{j}$ is denoted by $L\left(H, V_{j}\right)$. Define

$$
\bigoplus_{j \in J} V_{j}=\left\{\left\{a_{j}\right\}_{j \in J}: a_{j} \in V_{j},\left\|\left\{a_{j}\right\}_{j \in J}\right\|^{2}=\sum_{j \in J}\left\|a_{j}\right\|^{2}<\infty\right\} .
$$

Then $\bigoplus_{j \in J} V_{j}$ is a Hilbert space under the inner product

$$
\left\langle\left\{a_{j}\right\}_{j \in J},\left\{b_{j}\right\}_{j \in J}\right\rangle=\sum_{j \in J}\left\langle a_{j}, b_{j}\right\rangle \quad \text { for }\left\{a_{j}\right\}_{j \in J},\left\{b_{j}\right\}_{j \in J} \in \bigoplus_{j \in J} V_{j}
$$

Suppose $\left\{e_{j, k}\right\}_{k \in K_{j}}$ is an orthonormal basis (simply o.n.b.) for $V_{j}$, where $K_{j} \subset \mathbb{Z}, j \in J$. Define $\tilde{e}_{j, k}=\left\{\delta_{j, i} e_{i, k}\right\}_{i \in J}$, where $\delta$ is the Kronecker symbol. Then $\left\{\tilde{e}_{j, k}\right\}_{j \in J, k \in K_{j}}$ is an o.n.b. for $\bigoplus_{j \in J} V_{j}$ (see [17]).

Definition 2.1 ([14]) A sequence $\left\{\Lambda_{j} \in L\left(H, V_{j}\right)\right\}_{j \in J}$ is called a g-frame for $H$ with respect to (w.r.t.) $\left\{V_{j}\right\}_{j \in J}$ if

$$
A\|f\|^{2} \leq \sum_{j \in J}\left\|\Lambda_{j} f\right\|^{2} \leq B\|f\|^{2}
$$


for all $f \in H$ and some positive constants $A \leq B$. The numbers $A, B$ are called the frame bounds. If only the right-hand inequality of $(2.1)$ is satisfied, then $\left\{\Lambda_{j}\right\}_{j \in J}$ is called a g-Bessel sequence for $H$ w.r.t. $\left\{V_{j}\right\}_{j \in J}$ with bound $B$. If $A=B=\lambda$, then $\left\{\Lambda_{j}\right\}_{j \in J}$ is called a $\lambda$-tight gframe. In addition, if $\lambda=1$, then $\left\{\Lambda_{j}\right\}_{j \in J}$ is called a Parsevel g-frame.

For a g-Bessel sequence $\left\{\Lambda_{j}\right\}_{j \in J}$ with bound $B$, the operator

$$
T_{\Lambda}: \bigoplus_{j \in J} V_{j} \rightarrow H, \quad T_{\Lambda} F=\sum_{j \in J} \Lambda_{j}^{*} f_{j}, \quad \forall F=\left\{f_{j}\right\}_{j \in J} \in \bigoplus_{j \in J} V_{j}
$$

is well-defined, and its adjoint is given by

$$
T_{\Lambda}^{*}: H \rightarrow \bigoplus_{j \in J} V_{j}, \quad T_{\Lambda}^{*} f=\left\{\Lambda_{j} f\right\}_{j \in J}, \quad \forall f \in H
$$

The operator $T_{\Lambda}$ is called the synthesis operator, and $T_{\Lambda}^{*}$ is called the analysis operator of $\left\{\Lambda_{j}\right\}_{j \in J}$. For g-frame $\left\{\Lambda_{j}\right\}_{j \in J}$ with bounds $A$ and $B$, the operator

$$
S_{\Lambda}: H \rightarrow H, \quad S_{\Lambda} f=\sum_{j \in J} \Lambda_{j}^{*} \Lambda_{j} f, \quad \forall f \in H
$$

is called a g-frame operator of $\left\{\Lambda_{j}\right\}_{j \in J}$. It is bounded, invertible, self-adjoint, and positive, and $A I_{H} \leq S_{\Lambda} \leq B I_{H}$. Let $\tilde{\Lambda}_{j}=\Lambda_{j} S_{\Lambda}^{-1}$. Then $\left\{\tilde{\Lambda}_{j}\right\}_{j \in J}$ is also a g-frame for $H$ w.r.t. $\left\{V_{j}\right\}_{j \in J}$ with the g-frame operator $S_{\Lambda}^{-1}$ and frame bounds $\frac{1}{B}$ and $\frac{1}{A} \cdot\left\{\tilde{\Lambda}_{j}\right\}_{j \in J}$ is called thebcanonical dual g-frame of $\left\{\Lambda_{j}\right\}_{j \in J}$ (see [14]).

Definition 2.2 ([14]) Let $\left\{\Lambda_{j}\right\}_{j \in J}$ be a g-frame for $H$ w.r.t. $\left\{V_{j}\right\}_{j \in J}$. A g-frame $\left\{\Gamma_{j}\right\}_{j \in J}$ is called an alternate dual $g$-frame for $\left\{\Lambda_{j}\right\}_{j \in J}$ if

$$
f=\sum_{j \in J} \Gamma_{j}^{*} \Lambda_{j} f, \quad \forall f \in H
$$

Moreover, $\left\{\Lambda_{j}\right\}_{j \in J}$ is also an alternate dual g-frame for $\left\{\Gamma_{j}\right\}_{j \in J}$, that is,

$$
f=\sum_{j \in J} \Lambda_{j}^{*} \Gamma_{j} f, \quad \forall f \in H
$$

Definition 2.3 ([20]) Let $\left\{f_{j}\right\}_{j \in J}$ and $\left\{g_{j}\right\}_{j \in J}$ be two Bessel sequences for $H$ with their respective synthesis operators $T_{f}$ and $T_{g}$. We say that $\left\{f_{j}\right\}_{j \in J}$ and $\left\{g_{j}\right\}_{j \in J}$ are approximately dual frames if $\left\|I_{H}-T_{f} T_{g}^{*}\right\|<1$ or $\left\|I_{H}-T_{g} T_{f}^{*}\right\|<1$.

It is clear that the operator $T_{f} T_{g}^{*}$ is invertible.

Definition 2.4 ([21]) Let $\left\{\Lambda_{j}\right\}_{j \in J}$ and $\left\{\Gamma_{j}\right\}_{j \in J}$ be two g-Bessel sequences for $H$ w.r.t. $\left\{V_{j}\right\}_{j \in J}$ with their respective synthesis operators $T_{\Lambda}$ and $T_{\Gamma}$. Then $\left\{\Lambda_{j}\right\}_{j \in J}$ and $\left\{\Gamma_{j}\right\}_{j \in J}$ are approximately dual g-frames if $\left\|I_{H}-T_{\Lambda} T_{\Gamma}^{*}\right\|<1$ or $\left\|I_{H}-T_{\Gamma} T_{\Lambda}^{*}\right\|<1$. 


\section{Dual and approximately dual g-frames}

This section focuses on the connection between approximately dual g-frames and dual g-frames and on a characterization of approximately dual g-frames.

Lemma 3.1 ([19]) Let $\left\{\Lambda_{j}\right\}_{j \in J}$ and $\left\{\Gamma_{j}\right\}_{j \in J}$ be two g-Bessel sequences for $H$ w.r.t. $\left\{V_{j}\right\}_{j \in J}$. Then the following are equivalent:

(i) $f=\sum_{j \in J} \Gamma_{j}^{*} \Lambda_{j} f, \forall f \in H$.

(ii) $f=\sum_{j \in J} \Lambda_{j}^{*} \Gamma_{j} f, \forall f \in H$.

(iii) $\langle f, g\rangle=\sum_{j \in J}\left\langle\Lambda_{j} f, \Gamma_{j} g\right\rangle, \forall f, g \in H$.

In case the equivalent conditions are satisfied, $\left\{\Lambda_{j}\right\}_{j \in J}$ and $\left\{\Gamma_{j}\right\}_{j \in J}$ are dual g-frames for $H$ w.r.t. $\left\{V_{j}\right\}_{j \in J}$.

Lemma 3.2 ([14]) Let $\Lambda_{j} \in L\left(H, V_{j}\right)$ for every $j \in J$, and let $\left\{e_{j, k}\right\}_{k \in K_{j}}$ be an o.n.b. for $V_{j}$. If $u_{j, k}$ is defined by $u_{j, k}=\Lambda_{j}^{*} e_{j, k}$, then $\left\{\Lambda_{j}\right\}_{j \in J}$ is a g-frame ( $g$-Bessel sequence) for $H$ if and only if $\left\{u_{j, k}\right\}_{j \in J, k \in K_{j}}$ is a frame (Bessel sequence) for $H$.

The following two theorems give a method to construct new dual g-frames (approximately dual g-frames) from given dual g-fromes.

Theorem 3.1 Let $\left\{\Lambda_{j}\right\}_{j \in J}$ and $\left\{\Gamma_{j}\right\}_{j \in J}$ be dual g-frames for $H$ w.r.t. $\left\{V_{j}\right\}_{j \in J}$, and let $O_{1}$ and $\mathrm{O}_{2}$ be two bounded operators on $H$ such that $\mathrm{O}_{2} O_{1}^{*}=I_{H}\left(\left\|I_{H}-O_{2} O_{1}^{*}\right\|<1\right)$. Then $\left\{\Lambda_{j} O_{1}\right\}_{j \in J}$ and $\left\{\Gamma_{j} \mathrm{O}_{2}\right\}_{j \in J}$ are dual g-frames (approximately dual g-frames) for $H$ w.r.t. $\left\{V_{j}\right\}_{j \in J}$.

Proof By a standard argument, $\left\{\Lambda_{j}\right\}_{j \in J}$ is a g-Bessel sequence with synthesis operator $T_{\Lambda}$. Since $O_{1}$ is a bounded operator on $H$, we see that $\left\{\Lambda_{j} O_{1}\right\}_{j \in J}$ is a g-Bessel sequence with synthesis operator $T_{O \Lambda}=O_{1} T_{\Lambda}$. Similarly, $\left\{\Gamma_{j} O_{2}\right\}_{j \in J}$ is also a g-Bessel sequence with synthesis operator $T_{O \Gamma}=O_{2} T_{\Gamma}$. By Lemma 3.1 we have

$$
\begin{aligned}
& T_{O \Gamma} T_{O \Lambda}^{*} f=O_{2} T_{\Gamma} T_{\Lambda}^{*} O_{1}^{*} f=O_{2} O_{1}^{*} f=f \\
& \left(\left\|I_{H}-T_{O \Gamma} T_{O \Lambda}^{*}\right\|=\left\|I_{H}-O_{2} T_{\Gamma} T_{\Lambda}^{*} O_{1}^{*}\right\|=\left\|I_{H}-O_{2} O_{1}^{*}\right\|<1\right)
\end{aligned}
$$

for all $f \in H$.

Corollary 3.1 Let $\left\{\Lambda_{j}\right\}_{j \in J}$ and $\left\{\Gamma_{j}\right\}_{j \in J}$ be dual g-frames for $H$ w.r.t. $\left\{V_{j}\right\}_{j \in J}$, and let $T$ be a unitary operator on $H$. Then $\left\{\Lambda_{j} T\right\}_{j \in J}$ and $\left\{\Gamma_{j} T\right\}_{j \in J}$ are dual g-frames (approximately dual g-frames) for $H$ w.r.t. $\left\{V_{j}\right\}_{j \in J}$.

Theorem 3.2 Assume that $\left\{\Lambda_{j}\right\}_{j \in J}$ and $\left\{\Gamma_{j}\right\}_{j \in J}$ are dual g-frames for $H$ w.r.t. $\left\{V_{j}\right\}_{j \in J}$, and let $\left\{\Lambda_{j}\right\}_{j \in J}$ and $\left\{\Delta_{j}\right\}_{j \in J}$ also be dual g-frames for $H$ w.r.t. $\left\{V_{j}\right\}_{j \in J}$. Then for any $\alpha \in \mathbb{C},\left\{\Lambda_{j}\right\}_{j \in J}$ and $\left\{\alpha \Gamma_{j}+(1-\alpha) \Delta_{j}\right\}_{j \in J}$ are dual g-frames for $H$ w.r.t. $\left\{V_{j}\right\}_{j \in J}$.

Proof By a standard argument, $\left\{\alpha \Gamma_{j}+(1-\alpha) \Delta_{j}\right\}_{j \in J}$ is a g-Bessel sequence for $H$ w.r.t. $\left\{V_{j}\right\}_{j \in J}$. By Lemma 3.1 we have

$$
\begin{aligned}
\sum_{j \in J}\left\langle\Lambda_{j} f,\left(\alpha \Gamma_{j}+(1-\alpha) \Delta_{j}\right) g\right\rangle & =\sum_{j \in J}\left\langle\Lambda_{j} f, \alpha \Gamma_{j} g\right\rangle+\sum_{j \in J}\left\langle\Lambda_{j} f,(1-\alpha) \Delta_{j} g\right\rangle \\
& =\bar{\alpha} \sum_{j \in J}\left\langle\Lambda_{j} f, \Gamma_{j} g\right\rangle+(1-\bar{\alpha}) \sum_{j \in J}\left\langle\Lambda_{j} f, \Delta_{j} g\right\rangle
\end{aligned}
$$




$$
\begin{aligned}
& =\bar{\alpha}\langle f, g\rangle+(1-\bar{\alpha})\langle f, g\rangle \\
& =\langle f, g\rangle
\end{aligned}
$$

for all $f, g \in H$.

Obviously, if $\left\{\Lambda_{j}\right\}_{j \in J}$ and $\left\{\Gamma_{j}\right\}_{j \in J}$ are dual g-frames for $H$ w.r.t. $\left\{V_{j}\right\}_{j \in J}$, then $\left\{\Lambda_{j}\right\}_{j \in J}$ and $\left\{\Gamma_{j}\right\}_{j \in J}$ are approximately dual g-frames for $H$ w.r.t. $\left\{V_{j}\right\}_{j \in J}$. However, the converse is not true in general. The following theorem gives a sufficient condition for approximately dual g-frames to be dual g-frames.

Theorem 3.3 Let $\left\{\Lambda_{j}\right\}_{j \in J}$ and $\left\{\Gamma_{j}\right\}_{j \in J}$ be approximately dual g-frames for $H$ w.r.t. $\left\{V_{j}\right\}_{j \in J}$ with synthesis operators $T_{\Lambda}$ and $T_{\Gamma}$, respectively. Then $T_{\Lambda} T_{\Gamma}^{*}$ is invertible; furthermore, the sequences $\left\{\Lambda_{j}\right\}_{j \in J}$ and $\left\{\left(T_{\Lambda} T_{\Gamma}^{*}\right)^{-1} \Gamma_{j}\right\}_{j \in J}$ are dual g-frames.

Proof Since $\left\{\Lambda_{j}\right\}_{j \in J}$ and $\left\{\Gamma_{j}\right\}_{j \in J}$ are approximately dual g-frames for $H$ w.r.t. $\left\{V_{j}\right\}_{j \in J}$, we have $\left\|I_{U}-T_{\Lambda} T_{\Gamma}^{*}\right\|<1$, and thus $T_{\Lambda} T_{\Gamma}^{*}$ is invertible on $H$. By Lemma 3.1 we have

$$
\begin{aligned}
\langle f, g\rangle & =\left\langle\left(T_{\Lambda} T_{\Gamma}^{*}\right)\left(T_{\Lambda} T_{\Gamma}^{*}\right)^{-1} f, g\right\rangle \\
& =\left\langle T_{\Gamma}^{*}\left(T_{\Lambda} T_{\Gamma}^{*}\right)^{-1} f, T_{\Lambda}^{*} g\right\rangle \\
& =\sum_{j \in J}\left\langle\Gamma_{j}\left(T_{\Lambda} T_{\Gamma}^{*}\right)^{-1} f, \Lambda_{j} g\right\rangle
\end{aligned}
$$

for all $f, g \in H$.

For Theorem 3.3, a natural question is whether a g-frame always corresponds to an approximately dual g-frame. The following theorem gives an affirmative answer.

Theorem 3.4 Let $\left\{\Lambda_{j}\right\}_{j \in J}$ be a g-frame for $H$ w.r.t. $\left\{V_{j}\right\}_{j \in J}$ with the synthesis operator $T_{\Lambda}$ and frame bounds $A$ and $B$. Then $\left\{B^{-1} \Lambda_{j}\right\}_{j \in J}$ is an approximately dual g-frame of $\left\{\Lambda_{j}\right\}_{j \in J}$.

Proof Note that $\left\{\Lambda_{j}\right\}_{j \in J}$ is a g-frame for $H$ w.r.t. $\left\{V_{j}\right\}_{j \in J}$ and $T_{\Lambda}$ is its synthesis operator. So $\left\{B^{-1} \Lambda_{j}\right\}_{j \in J}$ is also g-frame with synthesis operator $B^{-1} T_{\Lambda}$, and

$$
\begin{aligned}
\left\|I_{H}-B^{-1} T_{\Lambda} T_{\Lambda}^{*}\right\| & =\sup _{\|f\|=1}\left|\left\langle\left(I_{H}-B^{-1} T_{\Lambda} T_{\Lambda}^{*}\right) f, f\right\rangle\right| \\
& \leq \frac{B-A}{B}<1 .
\end{aligned}
$$

It follows that $\left\{B^{-1} \Lambda_{j}\right\}_{j \in J}$ is an approximately dual g-frame of $\left\{\Lambda_{j}\right\}_{j \in J}$.

From Theorem 3.4 we know that every g-frame has at least an approximately dual gframe. Next, we characterize all approximately dual g-frames for a given g-frame. For this purpose, we need to establish some lemmas.

Lemma 3.3 Let $\left\{\Lambda_{j}\right\}_{j \in J}$ be a g-frame for $H$ w.r.t. $\left\{V_{j}\right\}_{j \in J}$, let $T_{\Lambda}$ be its synthesis operator, and let $\left\{\tilde{e}_{j, k}\right\}_{j \in J, k \in K_{j}}$ be an o.n.b. for $\bigoplus_{j \in J} V_{j}$. Then $\left\{\Gamma_{j}\right\}_{j \in J}$ and $\left\{\Lambda_{j}\right\}_{j \in J}$ are approximately dual $g$-frames if and only if $\Gamma_{j}^{*} e_{j, k}=T \tilde{e}_{j, k}\left(\forall j \in J, k \in K_{j}\right)$, where $T: \bigoplus_{j \in J} V_{j} \rightarrow H$ is a linear bounded operator such that $\left\|I_{H}-T T_{\Lambda}^{*}\right\|<1$. 
Proof Necessity. Suppose $\left\{\Gamma_{j}\right\}_{j \in J}$ is an approximately dual g-frame of $\left\{\Lambda_{j}\right\}_{j \in J}$. Then $\left\{\Gamma_{j}\right\}_{j \in J}$ is a g-frame, and $\left\|I_{H}-T_{\Gamma} T_{\Lambda}^{*}\right\|<1$, where $T_{\Gamma}$ is the synthesis operator of $\left\{\Gamma_{j}\right\}_{j \in J}$. Notice that

$$
T_{\Gamma} \tilde{e}_{j, k}=T_{\Gamma}\left(\left\{\delta_{j, i} e_{i, k}\right\}_{i \in J}\right)=\sum_{i \in J} \Gamma_{j}^{*} \delta_{j, i} e_{i, k}=\Gamma_{j}^{*} e_{j, k}
$$

Denote $T=T_{\Gamma}$. Then $T: \bigoplus_{j \in J} V_{j} \rightarrow H$ is a linear bounded operator satisfying $\left\|I_{H}-T T_{\Lambda}^{*}\right\|<1$ and $\Gamma_{j}^{*} e_{j, k}=T \tilde{e}_{j, k}$ for $j \in J, k \in K_{j}$.

Next, we prove the converse. Suppose $T: \bigoplus_{j \in J} V_{j} \rightarrow H$ is a linear bounded operator satisfying $\left\|I_{H}-T T_{\Lambda}^{*}\right\|<1$ and $\Gamma_{j}^{*} e_{j, k}=T \tilde{e}_{j, k}$ for $j \in J, k \in K_{j}$. Then

$$
\begin{aligned}
T T_{\Lambda}^{*} f & =T\left(\left\{\Lambda_{j} f\right\}_{j \in J}\right) \\
& =T\left(\sum_{j \in J} \sum_{k \in K_{j}}\left\langle\Lambda_{j} f, e_{j, k}\right) \tilde{e}_{j, k}\right) \\
& =\sum_{j \in J} \sum_{k \in K_{j}}\left\langle\Lambda_{j} f, e_{j, k}\right\rangle T \tilde{e}_{j, k} \\
& =\sum_{j \in J} \sum_{k \in K_{j}}\left\langle\Lambda_{j} f, e_{j, k}\right\rangle \Gamma_{j}^{*} e_{j, k} \\
& =\sum_{j \in J} \Gamma_{j}^{*} \sum_{k \in K_{j}}\left\langle\Lambda_{j} f, e_{j, k}\right\rangle e_{j, k} \\
& =\sum_{j \in J} \Gamma_{j}^{*} \Lambda_{j} f
\end{aligned}
$$

for $f \in H$. Since $\left\{\tilde{e}_{j, k}\right\}_{j \in J, k \in K_{j}}$ is an o.n.b. for $\bigoplus_{j \in J} V_{j}$, we have that $\left\{T \tilde{e}_{j, k}\right\}_{j \in J, k \in K_{j}}$ is a Bessel sequence for $H$. Let $u_{j, k}=T \tilde{e}_{j, k}$. Then $u_{j, k}=\Gamma_{j}^{*} e_{j, k}$. By Lemma $3.2\left\{\Gamma_{j}\right\}_{j \in J}$ is a g-Bessel sequence for $H$ w.r.t. $\left\{V_{j}\right\}_{j \in J}$. Let $T_{\Gamma}$ be the synthesis operator of $\left\{\Gamma_{j}\right\}_{j \in J}$. Then $T=T_{\Gamma}$ and $\left\|I_{H}-T_{\Gamma} T_{\Lambda}^{*}\right\|<1$, and hence $\left\{\Gamma_{j}\right\}_{j \in J}$ and $\left\{\Lambda_{j}\right\}_{j \in J}$ are approximately dual g-frames.

From Lemma 3.3 we know that $T$ is very important. The following lemma gives an explicit expression of $T$ in Lemma 3.3.

Lemma 3.4 Let $\left\{\Lambda_{j}\right\}_{j \in J}$ be a g-frame for $H$ w.r.t. $\left\{V_{j}\right\}_{j \in J}$ with the synthesis operator $T_{\Lambda}$ and the frame operator $S_{\Lambda}$. Then $\left\|I_{H}-T T_{\Lambda}^{*}\right\|<1\left(T: \bigoplus_{j \in J} V_{j} \rightarrow H\right)$ if and only if $T=S_{\Lambda}^{-1} T_{\Lambda}+$ $W\left(I-T_{\Lambda}^{*} Q S_{\Lambda}^{-1} T_{\Lambda}\right)$, where I is the identity operator on $\bigoplus_{j \in J} V_{j}$, and $W: \bigoplus_{j \in J} V_{j} \rightarrow H$ and $Q: H \rightarrow H$ are linear bounded operators satisfying $\left\|W T_{\Lambda}^{*}\left(I_{H}-Q\right)\right\|<1$.

Proof First, we suppose that $\left\|I_{H}-T T_{\Lambda}^{*}\right\|<1\left(T \in L\left(\bigoplus_{j \in J} V_{j}, H\right)\right)$. Then $T T_{\Lambda}^{*}$ is invertible. Let $W=T$ and $Q=\left(T T_{\Lambda}^{*}\right)^{-1}$. Then

$$
\begin{aligned}
S_{\Lambda}^{-1} T_{\Lambda}+W\left(I-T_{\Lambda}^{*} Q S_{\Lambda}^{-1} T_{\Lambda}\right) & =S_{\Lambda}^{-1} T_{\Lambda}+T\left(I-T_{\Lambda}^{*}\left(T T_{\Lambda}^{*}\right)^{-1} S_{\Lambda}^{-1} T_{\Lambda}\right) \\
& =S_{\Lambda}^{-1} T_{\Lambda}+T-T T_{\Lambda}^{*}\left(T T_{\Lambda}^{*}\right)^{-1} S_{\Lambda}^{-1} T_{\Lambda} \\
& =S_{\Lambda}^{-1} T_{\Lambda}+T-S_{\Lambda}^{-1} T_{\Lambda}=T .
\end{aligned}
$$


Conversely, assume that $T=S_{\Lambda}^{-1} T_{\Lambda}+W\left(I-T_{\Lambda}^{*} Q S_{\Lambda}^{-1} T_{\Lambda}\right)$. Then

$$
\begin{aligned}
T T_{\Lambda}^{*} & =\left(S_{\Lambda}^{-1} T_{\Lambda}+W\left(I-T_{\Lambda}^{*} Q S_{\Lambda}^{-1} T_{\Lambda}\right)\right) T_{\Lambda}^{*} \\
& =S_{\Lambda}^{-1} T_{\Lambda} T_{\Lambda}^{*}+W T_{\Lambda}^{*}-W T_{\Lambda}^{*} Q S_{\Lambda}^{-1} T_{\Lambda} T_{\Lambda}^{*} \\
& =I_{U}+W T_{\Lambda}^{*}-W T_{\Lambda}^{*} Q .
\end{aligned}
$$

Therefore

$$
\left\|I_{H}-T T_{\Lambda}^{*}\right\|=\left\|W T_{\Lambda}^{*}\left(I_{H}-Q\right)\right\|<1
$$

Now, we turn to characterizing all approximately dual g-frames for a given g-frame.

Theorem 3.5 Let $\left\{\Gamma_{j} \in L\left(H, V_{j}\right)\right\}$ be a sequence, and let $\left\{\Lambda_{j}\right\}_{j \in J}$ be a g-frame for $H$ w.r.t. $\left\{V_{j}\right\}_{j \in J}$ with the synthesis operator $T_{\Lambda}$ and the frame operator $S_{\Lambda}$. Then $\left\{\Lambda_{j}\right\}_{j \in J}$ and $\left\{\Gamma_{j}\right\}_{j \in J}$ are approximately dual $g$-frames if and only if

$$
\Gamma_{j}^{*} e_{j, k}=S_{\Lambda}^{-1} \Lambda_{j}^{*} e_{j, k}+W \tilde{e}_{j, k}-\sum_{j^{\prime} \in J} \sum_{k^{\prime} \in K_{j}}\left\langle Q S_{\Lambda}^{-1} \Lambda_{j}^{*} e_{j, k}, \Lambda_{j^{\prime}}^{*} e_{j^{\prime}, k^{\prime}}\right\rangle W \tilde{e}_{j^{\prime}, k^{\prime}}, \quad \forall j \in J, k \in K_{j},
$$

where $W: \bigoplus_{j \in J} V_{j} \rightarrow H$ and $Q: H \rightarrow H$ are linear bounded operators satisfying $\left\|W T_{\Lambda}^{*}\left(I_{H}-Q\right)\right\|<1$.

Proof First, we assume that $\left\{\Lambda_{j}\right\}_{j \in J}$ and $\left\{\Gamma_{j}\right\}_{j \in J}$ are approximately dual g-frames. By Lemmas 3.3 and 3.4 we have

$$
\Gamma_{j}^{*} e_{j, k}=\left(S_{\Lambda}^{-1} T_{\Lambda}+W\left(I-T_{\Lambda}^{*} Q S_{\Lambda}^{-1} T_{\Lambda}\right)\right) \tilde{e}_{j, k},
$$

where $I$ is the identity operator on $\bigoplus_{j \in J} V_{j}$, and $W: \bigoplus_{j \in J} V_{j} \rightarrow H$ and $Q: H \rightarrow H$ are linear bounded operators satisfying $\left\|W T_{\Lambda}^{*}\left(I_{U}-Q\right)\right\|<1$. Set $z_{j, k}=W \tilde{e}_{j, k}$. We know that $\left\{z_{j, k}\right\}_{j \in J, k \in K_{j}}$ is a Bessel sequence for $H$. Using the notations $u_{j, k}:=\Lambda_{j}^{*} e_{j, k}$ and $v_{j, k}:=\Gamma_{j}^{*} e_{j, k}$, we have

$$
\left\{\left\langle Q S_{\Lambda}^{-1} u_{j, k}, u_{j^{\prime}, k^{\prime}}\right\rangle\right\}_{j^{\prime} \in J, k^{\prime} \in K_{j}} \in l^{2}
$$

for any $j \in J$ and $k \in K_{j}$. So $\sum_{j^{\prime} \in J} \sum_{k^{\prime} \in K_{j}}\left\langle Q S_{\Lambda}^{-1} u_{j, k}, u_{j^{\prime}, k^{\prime}}\right\rangle z_{j^{\prime}, k^{\prime}}$ converges unconditionally. By (3.2) we have

$$
\begin{aligned}
v_{j, k} & =S_{\Lambda}^{-1} T_{\Lambda} \tilde{e}_{j, k}+W \tilde{e}_{j, k}-W T_{\Lambda}^{*} Q S_{\Lambda}^{-1} T_{\Lambda} \tilde{e}_{j, k} \\
& =S_{\Lambda}^{-1} u_{j, k}+z_{j, k}-W T_{\Lambda}^{*} Q S_{\Lambda}^{-1} u_{j, k} \\
& =S_{\Lambda}^{-1} u_{j, k}+z_{j, k}-W\left(\sum_{j^{\prime} \in J} \sum_{k^{\prime} \in K_{j}}\left\langle\Lambda_{j^{\prime}} Q S_{\Lambda}^{-1} u_{j, k}, e_{j^{\prime}, k^{\prime}}\right\rangle \tilde{e}_{j^{\prime}, k^{\prime}}\right) \\
& =S_{\Lambda}^{-1} u_{j, k}+z_{j, k}-\sum_{j^{\prime} \in J} \sum_{k^{\prime} \in K_{j}}\left\langle Q S_{\Lambda}^{-1} u_{j, k}, \Lambda_{j^{\prime}}^{*} e_{j^{\prime}, k^{\prime}}\right\rangle W \tilde{e}_{j^{\prime}, k^{\prime}} \\
& =S_{\Lambda}^{-1} u_{j, k}+z_{j, k}-\sum_{j^{\prime} \in J} \sum_{k^{\prime} \in K_{j}}\left\langle Q S_{\Lambda}^{-1} u_{j, k}, u_{j^{\prime}, k^{\prime}}\right| z_{j^{\prime}, k^{\prime}},
\end{aligned}
$$


that is,

$$
\Gamma_{j}^{*} e_{j, k}=S_{\Lambda}^{-1} \Lambda_{j}^{*} e_{j, k}+W \tilde{e}_{j, k}-\sum_{j^{\prime} \in J} \sum_{k^{\prime} \in K_{j}}\left\langle Q S_{\Lambda}^{-1} \Lambda_{j}^{*} e_{j, k}, \Lambda_{j^{\prime}}^{*} e_{j^{\prime}, k^{\prime}}\right\rangle W \tilde{e}_{j^{\prime}, k^{\prime}}
$$

for all $j \in J, k \in K_{j}$.

Now we prove the converse. Assume that (3.1) holds. For any $f \in H$, using the notations $u_{j, k}:=\Lambda_{j}^{*} e_{j, k}, v_{j, k}:=\Gamma_{j}^{*} e_{j, k}$, and $z_{j, k}:=W \tilde{e}_{j, k}$, by a standard argument we get that $\sum_{j \in J} \sum_{k \in K_{j}}\left\langle f, u_{j, k}\right\rangle S_{\Lambda}^{-1} u_{j, k}$ converges unconditionally to $f$. Therefore

$$
\begin{aligned}
\sum_{j \in \mathcal{J}} \Gamma_{j}^{*} \Lambda_{j} f= & \sum_{j \in \mathcal{J}} \Gamma_{j}^{*} \sum_{k \in \mathcal{K}_{j}}\left\langle\Lambda_{j} f, e_{j, k}\right\rangle e_{j, k} \\
= & \sum_{j \in \mathcal{J}} \sum_{k \in \mathcal{K}_{j}}\left\langle f, \Lambda_{j}^{*} e_{j, k}\right\rangle \Gamma_{j}^{*} e_{j, k} \\
= & \sum_{j \in \mathcal{J}} \sum_{k \in \mathcal{K}_{j}}\left\langle f, u_{j, k}\right\rangle v_{j, k} \\
= & \sum_{j \in \mathcal{J}} \sum_{k \in \mathcal{K}_{j}}\left\langle f, u_{j, k}\right\rangle\left(S_{\Lambda}^{-1} u_{j, k}+z_{j, k}-\sum_{j^{\prime} \in \mathcal{J}} \sum_{k^{\prime} \in \mathcal{K}_{j}}\left\langle Q S_{\Lambda}^{-1} u_{j, k}, u_{j^{\prime}, k^{\prime}}\right\rangle z_{j^{\prime}, k^{\prime}}\right) \\
= & \sum_{j \in \mathcal{J}} \sum_{k \in \mathcal{K}_{j}}\left\langle f, u_{j, k}\right\rangle S_{\Lambda}^{-1} u_{j, k}+\sum_{j \in \mathcal{J}} \sum_{k \in \mathcal{K}_{j}}\left\langle f, u_{j, k}\right\rangle z_{j, k} \\
& -\sum_{j \in \mathcal{J}} \sum_{k \in \mathcal{K}_{j}}\left\langle f, u_{j, k}\right\rangle \sum_{j^{\prime} \in \mathcal{J}} \sum_{k^{\prime} \in \mathcal{K}_{j}}\left\langle Q S_{\Lambda}^{-1} u_{j, k}, u_{j^{\prime}, k^{\prime}}\right\rangle z_{j^{\prime}, k^{\prime}} \\
= & f+\sum_{j \in \mathcal{J}} \sum_{k \in \mathcal{K}_{j}}\left\langle f, u_{j, k}\right\rangle z_{j, k}-\sum_{j \in \mathcal{J}} \sum_{k \in \mathcal{K}_{j}}\left\langle Q \sum_{j^{\prime} \in \mathcal{J}} \sum_{k^{\prime} \in \mathcal{K}_{j}}\left\langle f, u_{j, k}\right\rangle S_{\Lambda}^{-1} u_{j, k}, u_{j^{\prime}, k^{\prime}}\right\rangle z_{j^{\prime}, k^{\prime}} \\
= & f+\sum_{j \in \mathcal{J}} \sum_{k \in \mathcal{K}_{j}}\left\langle f, u_{j, k}\right\rangle z_{j, k}-\sum_{j \in \mathcal{J}} \sum_{k \in \mathcal{K}_{j}}\left\langle Q f, u_{j^{\prime}, k^{\prime}}\right\rangle z_{j^{\prime}, k^{\prime}} \\
= & f+\sum_{j \in \mathcal{J}} \sum_{k \in \mathcal{K}_{j}}\left\langle f-Q f, u_{j, k}\right\rangle z_{j, k}
\end{aligned}
$$

for all $f \in H$. Next, we prove that $\left\{\Gamma_{j}\right\}_{j \in J}$ is a g-Bessel sequence for $H$ w.r.t. $\left\{V_{j}\right\}_{j \in J}$. Indeed,

$$
\begin{aligned}
\sum_{j \in J}\left\|\Gamma_{j} f\right\|^{2}= & \sum_{j \in \mathcal{J}} \sum_{k \in K_{j}}\left|\left\langle\Gamma_{j} f, e_{j, k}\right\rangle\right|^{2} \\
= & \sum_{j \in J} \sum_{k \in K_{j}}\left|\left\langle f, v_{j, k}\right\rangle\right|^{2} \\
= & \sum_{j \in J} \sum_{k \in K_{j}}\left|\left\langle f, S_{\Lambda}^{-1} u_{j, k}+z_{j, k}-\sum_{j^{\prime} \in J} \sum_{k^{\prime} \in K_{j}}\left\langle Q S_{\Lambda}^{-1} u_{j, k}, u_{j^{\prime}, k^{\prime}}\right\rangle z_{j^{\prime}, k^{\prime}}\right\rangle\right|^{2} \\
\leq & C_{1}\left(\sum_{j \in \mathcal{J}} \sum_{k \in \mathcal{K}_{j}}\left|\left\langle f, S_{\Lambda}^{-1} u_{j, k}\right\rangle\right|^{2}+\sum_{j \in J} \sum_{k \in K_{j}}\left|\left\langle f, z_{j, k}\right\rangle\right|^{2}\right. \\
& \left.+\sum_{j \in J} \sum_{k \in K_{j}}\left|\left\langle Q^{*} \sum_{j^{\prime} \in J} \sum_{k^{\prime} \in K_{j}}\left\langle f, z_{j^{\prime}, k^{\prime}}\right\rangle u_{j^{\prime}, k^{\prime}}, S_{\Lambda}^{-1} u_{j, k}\right\rangle\right|^{2}\right)
\end{aligned}
$$




$$
\begin{aligned}
& \leq C_{2}\left(\|f\|^{2}+\left\|Q^{*} \sum_{j^{\prime} \in J} \sum_{k^{\prime} \in K_{j}}\left\langle f, z_{j^{\prime}, k^{\prime}}\right\rangle u_{j^{\prime}, k^{\prime}}\right\|^{2}\right) \\
& \leq C_{3}\left(\|f\|^{2}+\sum_{j^{\prime} \in J} \sum_{k^{\prime} \in K_{j}}\left|\left\langle f, z_{j^{\prime} k^{\prime}}\right\rangle\right|^{2}\right) \\
& \leq C_{4}\|f\|^{2}
\end{aligned}
$$

for all $f \in H$, where $C_{1}, C_{2}, C_{3}$, and $C_{4}$ are different positive constants. Let $T_{\Gamma}$ be the synthesis operator of $\left\{\Gamma_{j}\right\}_{j \in J}$. Then

$$
\begin{aligned}
\left\|\left(I_{H}-T_{\Gamma} T_{\Lambda}^{*}\right) f\right\| & =\left\|\sum_{j \in J} \sum_{k \in K_{j}}\left\langle f-Q f, u_{j, k}\right\rangle z_{j, k}\right\| \\
& =\left\|\sum_{j \in J} \sum_{k \in K_{j}}\left\langle f-Q f, u_{j, k}\right\rangle W \tilde{e}_{j, k}\right\| \\
& =\left\|W \sum_{j \in J} \sum_{k \in K_{j}}\left\langle f-Q f, u_{j, k}\right\rangle \tilde{e}_{j, k}\right\| \\
& =\left\|W \sum_{j \in J} \sum_{k \in K_{j}}\left\langle\Lambda_{j}(f-Q f), e_{j, k}\right\rangle \tilde{e}_{j, k}\right\| \\
& =\left\|W T_{\Lambda}^{*}(f-Q f)\right\| \\
& \leq\left\|W T_{\Lambda}^{*}\left(I_{H}-Q\right)\right\|\|f\|
\end{aligned}
$$

for all $f \in H$. Therefore $\left\|I_{H}-T_{\Gamma} T_{\Lambda}^{*}\right\|<1$, and thus $\left\{\Lambda_{j}\right\}_{j \in J}$ and $\left\{\Gamma_{j}\right\}_{j \in J}$ are approximately dual g-frames.

\section{Perturbations of approximately dual g-frames}

The stability of frames is of great importance in frame theory, and it is studied widely by a lot of authors $([4,18])$. In this section, we show that, under some conditions, approximately dual g-frames and g-frames are stable under some perturbations. We first introduce some lemmas.

Lemma 4.1 ([17]) Let $\left\{\Lambda_{j}\right\}_{j \in J}$ be a g-frame for $H$ w.r.t. $\left\{V_{j}\right\}_{j \in J}$ with bounds $A$ and $B, \lambda_{1}, \lambda_{2} \in$ $(-1,1), \mu \geq 0$, and $\max \left\{\lambda_{1}+\frac{\mu}{\sqrt{A}}, \lambda_{2}\right\}<1$. If $\left\{\Gamma_{j} \in L\left(H, V_{j}\right)\right\}_{j \in J}$ satisfies

$$
\left\|\sum_{j \in J_{1}}\left(\Lambda_{j}-\Gamma_{j}\right)^{*} g_{j}\right\| \leq \lambda_{1}\left\|\sum_{j \in J_{1}} \Lambda_{j}^{*} g_{j}\right\|+\lambda_{2}\left\|\sum_{j \in J_{1}} \Gamma_{j}^{*} g_{j}\right\|+\mu\left(\sum_{j \in J_{1}}\left\|g_{j}\right\|^{2}\right)^{\frac{1}{2}}
$$

for an arbitrary finite subset $J_{1} \subset J$ and $g_{j} \in V_{j}$, then $\left\{\Gamma_{j}\right\}_{j \in J}$ is a g-frame for $H$ w.r.t. $\left\{V_{j}\right\}_{j \in J}$ with bounds

$$
\frac{\left(\left(1-\lambda_{1}\right) \sqrt{A}-\mu\right)^{2}}{\left(1+\lambda_{2}\right)^{2}}, \quad \frac{\left(\left(1+\lambda_{1}\right) \sqrt{B}+\mu\right)^{2}}{\left(1-\lambda_{2}\right)^{2}} .
$$


Lemma 4.2 ([14]) Let $\left\{\Lambda_{j}\right\}_{j \in J}$ be a g-frame for $H$ w.r.t. $\left\{V_{j}\right\}_{j \in J}$. Then for $g_{j} \in V_{j}$ satisfying $f=\sum_{j \in J} \Lambda_{j}^{*} g_{j}$, we have

$$
\sum_{j \in J}\left\|g_{j}\right\|^{2} \geq \sum_{j \in J}\left\|\tilde{\Lambda}_{j} f\right\|^{2}
$$

Lemma 4.3 ([14]) $\left\{\Lambda_{j}\right\}_{j \in J}$ is a $g$-Bessel sequence with an upper bound $B$ if and only if

$$
\left\|\sum_{j \in J_{1}} \Lambda_{j}^{*} g_{j}\right\|^{2} \leq B \sum_{j \in J_{1}}\left\|g_{j}\right\|^{2}, \quad g_{j} \in V_{j}
$$

where $J_{1}$ is an arbitrary finite subset of $J$.

Theorem 4.1 Let $\Lambda_{j} \in L\left(H, V_{j}\right)$, let $\left\{\Gamma_{j}\right\}_{j \in J}$ be a g-frame for $H$ w.r.t. $\left\{V_{j}\right\}_{j \in J}$ with bounds $A$ and $B$ and the synthesis operator $T_{\Lambda}$, and let $\left\{\Delta_{j}\right\}_{j \in J}$ be alternate dual for $\left\{\Gamma_{j}\right\}_{j \in J}$ with the upper bound $C$ and the synthesis operator $T_{\Delta}$. Assume that there are constants $\lambda_{1}, \mu \geq 0$, and $0 \leq \lambda_{2}<1$ satisfying

$$
\left\|\sum_{j \in J_{1}}\left(\Gamma_{j}-\Lambda_{j}\right)^{*} g_{j}\right\| \leq \lambda_{1}\left\|\sum_{j \in J_{1}} \Gamma_{j}^{*} g_{j}\right\|+\lambda_{2}\left\|\sum_{j \in J_{1}} \Lambda_{j}^{*} g_{j}\right\|+\mu\left(\sum_{j \in J_{1}}\left\|g_{j}\right\|^{2}\right)^{\frac{1}{2}},
$$

where $J_{1}$ is an arbitrary finite subset of $J$, and $g_{j} \in V_{j}$. If

$$
\lambda_{1}+\lambda_{2} \sqrt{B C}\left(1+\frac{\lambda_{1}+\lambda_{2}+\frac{\mu}{\sqrt{B}}}{1-\lambda_{2}}\right)+\mu \sqrt{C}<1
$$

then $\left\{\Lambda_{j}\right\}_{j \in J}$ and $\left\{\Delta_{j}\right\}_{j \in J}$ are approximately dual g-frames.

Proof By Lemma 4.2 we have $C \geq \frac{1}{A}$ and $B C \geq \frac{B}{A} \geq 1$. Note that

$$
\lambda_{1}+\lambda_{2} \sqrt{B C}\left(1+\frac{\lambda_{1}+\lambda_{2}+\frac{\mu}{\sqrt{B}}}{1-\lambda_{2}}\right)+\mu \sqrt{C}<1 .
$$

It follows that $\lambda_{1}+\frac{\mu}{\sqrt{A}}<1$. By Lemma $4.1\left\{\Lambda_{j}\right\}_{j \in J}$ is a g-frame for $H$ w.r.t. $\left\{V_{j}\right\}_{j \in J}$ with bounds

$$
A\left(1-\frac{\lambda_{1}+\lambda_{2}+\frac{\mu}{\sqrt{A}}}{1+\lambda_{2}}\right)^{2}, \quad B\left(1+\frac{\lambda_{1}+\lambda_{2}+\frac{\mu}{\sqrt{B}}}{1-\lambda_{2}}\right)^{2}
$$

Denote by $T_{\Lambda}$ the synthesis operator of $\left\{\Lambda_{j}\right\}_{j \in J}$. From (4.1) we have

$$
\left\|T_{\Gamma} c-T_{\Lambda} c\right\| \leq \lambda_{1}\left\|T_{\Gamma} c\right\|+\lambda_{2}\left\|T_{\Lambda} c\right\|+\mu\|c\|_{\oplus_{j \in J} V_{j}}
$$

for any $c=\left\{c_{j}\right\}_{j \in J} \in \bigoplus_{j \in J} V_{j}$. Take $c=T_{\Delta}^{*} f$ in (4.2). Then

$$
\begin{aligned}
\left\|\left(I_{H}-T_{\Lambda} T_{\Delta}^{*}\right) f\right\| & \leq \lambda_{1}\|f\|+\lambda_{2}\left\|T_{\Lambda} T_{\Delta}^{*} f\right\|+\mu\left\|T_{\Delta}^{*} f\right\|_{\bigoplus_{j \in J} V_{j}} \\
& \leq \lambda_{1}\|f\|+\lambda_{2} \sqrt{C}\left\|T_{\Lambda}\right\|\|f\|+\mu \sqrt{C}\|f\|
\end{aligned}
$$




$$
\begin{aligned}
& \leq \lambda_{1}\|f\|+\lambda_{2} \sqrt{B C}\left(1+\frac{\lambda_{1}+\lambda_{2}+\frac{\mu}{\sqrt{B}}}{1-\lambda_{2}}\right)\|f\|+\mu \sqrt{C}\|f\| \\
& =\left(\lambda_{1}+\lambda_{2} \sqrt{B C}\left(1+\frac{\lambda_{1}+\lambda_{2}+\frac{\mu}{\sqrt{B}}}{1-\lambda_{2}}\right)+\mu \sqrt{C}\right)\|f\|
\end{aligned}
$$

for any $f \in H$. So

$$
\left\|I_{H}-T_{\Lambda} T_{\Delta}^{*}\right\| \leq \lambda_{1}+\lambda_{2} \sqrt{B C}\left(1+\frac{\lambda_{1}+\lambda_{2}+\frac{\mu}{\sqrt{B}}}{1-\lambda_{2}}\right)+\mu \sqrt{C}<1 .
$$

Thus $\left\{\Lambda_{j}\right\}_{j \in J}$ and $\left\{\Delta_{j}\right\}_{j \in J}$ are approximately dual g-frames if $\lambda_{1}+\lambda_{2} \sqrt{B C}\left(1+\frac{\lambda_{1}+\lambda_{2}+\frac{\mu}{\sqrt{B}}}{1-\lambda_{2}}\right)+$ $\mu \sqrt{C}<1$.

From Theorem 4.1 we can obtain immediately the following corollary.

Corollary 4.1 Let $\Lambda_{j} \in L\left(H, V_{j}\right)$, let $\left\{\Gamma_{j}\right\}_{j \in J}$ be a g-frame for $H$ w.r.t. $\left\{V_{j}\right\}_{j \in J}$ with bounds $A$ and $B$ and the synthesis operator $T_{\Gamma}$, and let $\left\{\Delta_{j}\right\}_{j \in J}$ be the canonical dual for $\left\{\Gamma_{j}\right\}_{j \in J}$ with the synthesis operator $T_{\Delta}$. Suppose that there are constants $\lambda_{1}, \mu \geq 0$, and $0 \leq \lambda_{2}<1$ such that

$$
\left\|\sum_{j \in J_{1}}\left(\Gamma_{j}-\Lambda_{j}\right)^{*} g_{j}\right\| \leq \lambda_{1}\left\|\sum_{j \in J_{1}} \Gamma_{j}^{*} g_{j}\right\|+\lambda_{2}\left\|\sum_{j \in J_{1}} \Lambda_{j}^{*} g_{j}\right\|+\mu\left(\sum_{j \in J_{1}}\left\|g_{j}\right\|^{2}\right)^{\frac{1}{2}},
$$

where $J_{1}$ is an arbitrary finite subset of $J$, and $g_{j} \in V_{j}$. If $\lambda_{1}+\lambda_{2} \sqrt{\frac{B}{A}}\left(1+\frac{\lambda_{1}+\lambda_{2}+\frac{\mu}{\sqrt{B}}}{1-\lambda_{2}}\right)+\frac{\mu}{\sqrt{A}}<1$, then $\left\{\Lambda_{j}\right\}_{j \in J}$ and $\left\{\Delta_{j}\right\}_{j \in J}$ are approximately dual $g$-frames.

Note that $\left\{\Gamma_{j}\right\}_{j \in J}$ is a Parseval g-frame for $H$ w.r.t. $\left\{V_{j}\right\}_{j \in J}$. Then $\left\{\Gamma_{j}\right\}_{j \in J}$ is the canonical dual for itself. We have the following:

Corollary 4.2 Let $\Lambda_{j} \in L\left(H, V_{j}\right)$, and let $\left\{\Gamma_{j}\right\}_{j \in J}$ be a Parseval g-frame for $H$ w.r.t. $\left\{V_{j}\right\}_{j \in J}$. Assume that there are constants $\lambda, \mu \geq 0$ such that

$$
\left\|\sum_{j \in J_{1}}\left(\Gamma_{j}-\Lambda_{j}\right)^{*} g_{j}\right\| \leq \lambda\left\|\sum_{j \in J_{1}} \Gamma_{j}^{*} g_{j}\right\|+\mu\left(\sum_{j \in J_{1}}\left\|g_{j}\right\|^{2}\right)^{\frac{1}{2}}
$$

for an arbitrary finite subset $J_{1} \subset J$ and $g_{j} \in V_{j}$. If $\lambda+\mu<1$, then $\left\{\Lambda_{j}\right\}_{j \in J}$ and $\left\{\Gamma_{j}\right\}_{j \in J}$ are approximately dual g-frames.

Corollary 4.3 Let $\left\{\Lambda_{j} \in L\left(H, V_{j}\right)\right\}_{j \in J}$ be a sequence, and let $\left\{\Gamma_{j}\right\}_{j \in J}$ be a g-frame for $H$ w.r.t. $\left\{V_{j}\right\}_{j \in J}$. Also, let $\left\{\Delta_{j}\right\}_{j \in J}$ be an alternate dual for $\left\{\Gamma_{j}\right\}_{j \in J}$ with the upper bound $C$. If there exists a constant $R$ such that $C R<1$ and

$$
\sum_{j \in J}\left\|\left(\Gamma_{j}-\Lambda_{j}\right) f\right\|^{2} \leq R\|f\|^{2}, \quad \forall f \in H
$$

then $\left\{\Lambda_{j}\right\}_{j \in J}$ and $\left\{\Delta_{j}\right\}_{j \in J}$ are approximately dual g-frames. 
Proof Take $\lambda_{1}=\lambda_{2}=0$ and $\mu=\sqrt{R}$ in Theorem 4.1. From Lemma 4.3 we know that (4.1) is equivalent to (4.5). Since $C R<1$, we have that $\left\{\Lambda_{j}\right\}_{j \in J}$ and $\left\{\Delta_{j}\right\}_{j \in J}$ are approximately dual g-frames.

Remark 4.1 Corollary 4.1 and Corollary 4.3 are Proposition 3.10(i) and Theorem 3.1(i) in [21], respectively. They are particular cases of our Theorem 4.1.

Theorem 4.2 Let $\left\{\Lambda_{j}\right\}_{j \in J}$ be a g-frame for $H$ w.r.t. $\left\{V_{j}\right\}_{j \in J}$ with synthesis operator $T_{\Lambda}$ and bounds $A$ and B. Assume that $\Gamma_{j} \in L\left(H, V_{j}\right)$ for all $j \in J$ and there exist constants $\lambda_{1}, \lambda_{2}, \mu \geq$ 0 such that

$$
\left\|\sum_{j \in J_{1}}\left(\Lambda_{j}-\Gamma_{j}\right)^{*} g_{j}\right\| \leq \lambda_{1}\left\|\sum_{j \in J_{1}} \Lambda_{j}^{*} g_{j}\right\|+\lambda_{2}\left\|\sum_{j \in J_{1}} \Gamma_{j}^{*} g_{j}\right\|+\mu\left(\sum_{j \in J_{1}}\left\|g_{j}\right\|^{2}\right)^{\frac{1}{2}}
$$

for an arbitrary finite subset $J_{1} \subset J$ and $g_{j} \in V_{j}$. If $\lambda_{1}+\frac{\mu}{\sqrt{A}}<1$ and $\lambda_{2}+\left(\lambda_{1} \sqrt{\frac{B}{A}}+\frac{\mu}{\sqrt{A}}\right) \times$ $\frac{1+\lambda_{2}}{1-\left(\lambda_{1}+\frac{\mu}{\sqrt{A}}\right)}<1$, then $\left\{\Gamma_{j}\right\}_{j \in J}$ is a g-frame for $H$ w.r.t. $\left\{V_{j}\right\}_{j \in J}$, and $\left\{\tilde{\Gamma}_{j}\right\}_{j \in J}$ and $\left\{\Lambda_{j}\right\}_{j \in J}$ are approximately dual g-frames.

Proof We can prove the theorem by an argument similar to that of Theorem 4.1.

\section{Conclusions}

For a given frame, it is usually not easy to find a dual frame. The notion of approximately dual frames was introduced by Christensen in 2010. It is a generalization of dual frames. In this paper, on one hand, we obtain the link between approximately dual g-frames and dual g-frames and characterize approximately dual g-frames. On the other hand, we give stability results of approximately dual g-frames, which cover the results obtained by other authors.

\section{Acknowledgements}

The authors would like to thank the reviewers for their suggestions, which greatly improved the readability of this paper.

\section{Funding}

The paper is supported by the National Natural Science Foundation of China (Grant No. 11271037).

\section{Competing interests}

The authors declare that they have no competing interests.

\section{Authors' contributions}

Both authors contributed to each part of this work equally and read and approved the final manuscript.

\section{Author details}

${ }^{1}$ Department of Information Engineering, Henan Finance University, Zhengzhou, P.R. China. ${ }^{2}$ School of Mathematics and Information Sciences, Henan University of Economics and Law, Zhengzhou, P.R. China.

\section{Publisher's Note}

Springer Nature remains neutral with regard to jurisdictional claims in published maps and institutional affiliations.

Received: 4 May 2018 Accepted: 17 July 2018 Published online: 27 July 2018

\section{References}

1. Gabor, D.: Theory of communications. J. Inst. Electr. Eng. 93, 429-457 (1946)

2. Duffin, R.J., Schaeffer, A.C.: A class of nonharmonic Fourier series. Trans. Am. Math. Soc. 72, 341-366 (1952)

3. Daubechies, I., Grossmann, A., Meyer, Y.: Painless nonorthogonal expansions. J. Math. Phys. 27, 1271-1283 (1986)

4. Christensen, O.: An Introduction to Frames and Riesz Bases. Birkhäuser, Boston (2003) 
5. Zhang, W.: Dual and approximately dual Hilbert-Schmidt frames in Hilbert spaces. Results Math. 73(1), Article ID 4 (2018)

6. Heil, C.: A Basis Theory Primer. Birkhäuser, New York (2010)

7. Khosravi, A., Musazadeh, K.: Fusion frames and g-frames. J. Math. Anal. Appl. 342, 1068-1083 (2008)

8. Eldar, Y:: Sampling with arbitrary sampling and reconstruction spaces and oblique dual frame vectors. J. Fourier Anal. Appl. 9, 77-96 (2003)

9. Han, D., Sun, W.: Reconstruction of signals from frame coefficients with erasures at unknown locations. IEEE Trans. Inf. Theory 60(7), 4013-4025 (2014)

10. Feichtinger, H.G., Onchis, D.M., Wiesmeyr, C.: Construction of approximate dual wavelet frames. Adv. Comput. Math. 40, 273-282 (2014)

11. Javanshiri, H.: Some properties of approximately dual frames in Hilbert spaces. Results Math. 70(34), 475-485 (2016)

12. Christensen, O., Goh, S.S.: From dual pairs of Gabor frames to dual pairs of wavelet frames and vice versa. Appl. Comput. Harmon. Anal. 36, 198-214 (2014)

13. Casazza, P.G.: The art of frame theory. Taiwan. J. Math. 4(2), 129-201 (2000)

14. Sun, W.C.: G-frames and g-Riesz bases. J. Math. Anal. Appl. 322(1), 437-452 (2006)

15. Casazza, P.G., Kutyniok, G.: Frame of subspace. Contemp. Math. 345, 87-114 (2004)

16. Fornasier, M.: Quasi-orthogonal decompositions of structured frames. J. Math. Anal. Appl. 289, 180-199 (2004)

17. Zhu, Y.C.: Characterizations of g-frames and g-Riesz bases in Hilbert spaces. Acta Math. Sin. Engl. Ser. 24(10), 1727-1736 (2008)

18. Sun, W.C.: Stability of g-frames. J. Math. Anal. Appl. 326(2), 858-868 (2007)

19. Yang, X.H., Li, D.F.: Some new equalities and inequalities for $\mathrm{g}$-frames and their dual frames. Acta Math. Sin. Engl. Ser. 52(5), 1033-1040 (2009)

20. Christensen, O., Laugesen, R.S.: Approximately dual frame pairs in Hilbert spaces and applications to Gabor frames. Sampl. Theory Signal Image Process. 9, 77-90 (2011)

21. Khosravi, A., Morteza Azandaryani, M.: Approximate duality of g-frames in Hilbert spaces. Acta Math. Sci. 34(3), 639-652 (2014)

22. Morteza Azandaryani, M.: On the approximate duality of g-frames and fusion frames. UPB Sci. Bull. 79(2), 83-94 (2017)

23. Mirzaee Azandaryani, M., Khosravi, A.: Duals and approximate duals of g-frames in Hilbert spaces. J. Linear Topol. Algebra 4(4), 259-265 (2016)

\section{Submit your manuscript to a SpringerOpen ${ }^{\circ}$ journal and benefit from:}

- Convenient online submission

- Rigorous peer review

- Open access: articles freely available online

- High visibility within the field

- Retaining the copyright to your article

Submit your next manuscript at $\boldsymbol{\nabla}$ springeropen.com 